\title{
Correlation between Thyroid Endocrine Status, Change in Body Weight and Macular Thickness in Obese Patients
}

\author{
Teresa Esposito ${ }^{1,2^{*}}$, Bruno Varriale ${ }^{2}$, Carlo Irregolare ${ }^{3}$, Michele Lanza ${ }^{3}$, Angelica Perna ${ }^{1}$, Giuseppe Paolisso ${ }^{4}$ and Marcellino Monda ${ }^{1,2}$ \\ ${ }^{1}$ Seconda Università degli Studi di Napoli, Servizio di Dietetica, Napoli, Italy \\ ${ }^{2}$ Dipartimento di Medicina Sperimentale sez. F.Bottazzi, Seconda Università degli Studi di Napoli, Napoli, Italy \\ ${ }^{3}$ Dipartimento Multidisciplinare di Specialità Mediche, Chirurgiche e Odontoiatriche, Seconda Università di Napoli, Napoli, Italy \\ ${ }^{4}$ Dipartimento di Scienze Mediche, Chirurgiche, Neurologiche, Metaboliche e dell'Invecchiamento, Seconda Università degli Studi di Napoli, Napoli, Italy \\ *Corresponding author: Teresa Esposito MD, Seconda Università degli Studi di Napoli , Napoli (Italy), Tel: +390815667621; E-mail: teresa.esposito@unina2.it \\ Rec date: September 11, 2015 Acc date: September 24, 2015 Pub date: September 30, 2015
}

Copyright: (c) 2015 Esposito T, et al. This is an open-access article distributed under the terms of the Creative Commons Attribution License, which permits unrestricted use, distribution, and reproduction in any medium, provided the original author and source are credited.

\begin{abstract}
Objective: Study the co-relationship between thyroid status, obesity and macular thickness (MT) since from ophthalmic point of view, regulation of the aqueous-lipid balance resulted altered.

Methods: Obese patients (40), 20 males and 20 females, aging between 30-50 years have been enrolled in this study. All patients underwent to both biochemical and OTC evaluation.

Results: After a low-calorie diet, low fat and low salt, a remarkable weight change; weight fat mass (-17\%), lean body mass $(+9.0 \%)$, BMI $(-13 \%)$ and body weight $(-13 \%)$ were achieved. All blood parameters significantly lowered: uric acid $(-45 \%)$, bilirubin $(-46 \%)$, total cholesterol $(-25 \%)$, HDL $(+63 \%)$, LDL $(-17 \%)$, triglycerides $(-37 \%)$, and circulating glucose $(-13 \%)$. A significative reduction of FT3, FT4 and TSH has also been observed. The reduction of the MT was also significant $(-7.5 \%)$. Significant correlations between the MT detected pre- and post-treatment and lean mass $(p<0.005)$, BMI post-treatment $(p<0.011)$, BMI reduction $(p<0.022)$ and body weight reduction $(p<0.027)$. Finally, the same significant correlations are also present between the MT detected post-treatment and posttreatment lean mass $(p<0.007)$, post-treatment BMI $(p<0.007)$, BMI reduction $(p<0.045)$ and body weight reduction $(p<0.042)$.
\end{abstract}

Conclusions: There is a correlation between body and blood parameters variations, thyroid endocrine status and MT changes in obese patients undergone hypo caloric diet.

\section{Article Summary}

- Low calorie diet, low fat and low salt content, in obese patients can reduce the risk to develop retinal alterations.

- It could be important to introduce OCT evaluation in obese patients (BMI> 30) to better manage both general and ocular obesity related changes.

- This tool may be important also in early detection of macular alterations in obese patients not necessarily related to diabetes.

Keywords: Thyroid hormone status; Obesity; Body weight; Diet; Macular thickness

\section{Introduction}

The World Health Organization defines obesity an epidemic condition characterized by an increase in fat mass (change in body composition), resulting in a compromised state of health (impairment of bodily function). Since it is not the excess weight, but the excess of adipose mass (or rather its distribution) to determine the increased risk of morbidity and mortality, the diagnosis of obesity requires the quantification of fat mass, expressed as a percentage, by weight body [1].
The cut-off point of $30 \mathrm{~kg} / \mathrm{m}^{2}$ (Body Mass Index, BMI) identifies obesity [2]. Epidemiological studies have shown a clear correlation between central adiposity and hypertension, ischemic heart disease, type 2 diabetes and risk of mortality. Abdominal obesity is associated with an increase of visceral adipose tissue mass and this is related to the blood glucose and insulinemia in both sexes [3].

The classification of overweight and obesity by BMI was associated with CV to identify the RR of type 2 diabetes, hypertension and cardiovascular disease [2].

The bioelectrical impedance analysis (BIA) show the bioelectrical impedance [4]. This is inversely proportional to the water present in the tissues and to electrolytes dissolved in it, as well as to the section of the conductor, while it is directly proportional to the length. In addition, the energy balance is the difference between the energy introduced through food and energy expenditure (basal metabolic rate, $\mathrm{BMR}+$ diet induced thermogenesis, DIT+exercise, EEE). In obese in the dynamic phase of weight energy balance is positive $[2,4]$.

Vital processes taking place in the body require a continuous supply of energy, which is derived exclusively from the combustion of food. In the obese subject (metabolism baseline) MB is greater than the subject of normal weight of equal sex, age and body size, in relation to the expansion of the Fat Free Mass (FFM) that represents the component 
metabolically more active, but also to the contribution of mass fat which becomes significant. In obesity status, when the increase in weight has reached a plateau, the $\mathrm{MB}$, not significantly different compared to normal weight subjects [5]. This does not exclude the presence of differences during the dynamic phase of weight increase. Leaving aside the body function abnormalities present in secondary obesity, in which the clinical examination and medical history moving towards imaging studies and blood tests are necessary to confirm the diagnostic hypothesis, obesity is associated with numerous biological alterations, defined co-morbidities [6]. The evaluation of the bodily function in obese coincides with the screening of such co-morbidities [6].

Patients who enter into a state of obesity ( $>30 \mathrm{BMI}$ ) have metabolic syndrome. Metabolic syndrome is the name for a group of risk factors that raises risk for heart disease and other health problems, such as diabetes and stroke. One of the groups of the risk factors is the endocrine status of thyroid hormone. The term "metabolic" refers to the biochemical processes involved in the body's normal functioning. Risk factors are traits, conditions, or habits that increase your chance of developing a disease [1].

The macula is the central area of the retina, the more sensitive to light stimuli. This is the region with the highest density of photoreceptors (in this case mainly the cones), nerve cells photosensitive delegated to the transformation of the light energy into electrical impulses. The latter depart from the retina and travel through the optic nerve, finally reaching the brain (in particular in the occipital lobe, placed in the rear area of the head), where they are interpreted as visual information (images). The macula is the part of the retina responsible for the distinct vision and perception of details.

Micro-vascular anomalies have been proved to be related to macular thickening in non-diabetic patients measured with ocular coherence tomography (OCT) [7].

This is the first paper studying correlation among body and blood parameters changes versus macular thickness variation in obese patients after 3 months from starting a hypo caloric diet.

\section{Methods}

In the current study we enrolled a total of 40 patients, 20 males and 20 females, aged from 30 to 45 years. Obese patients have been enrolled in Dietetics unit and then evaluated in the Ophthalmology units of our university. All patients had increased levels of FT3, FT4 and TSH. All treatments follow the guideline of our Comitato Etico dell'Azienda Ospedaliera Universitaria of the Second University of Naples. The study was performed in accordance with the ethical standards stated in the 1964 Declaration of Helsinki and approved by the local clinical research ethics committee (protocol number 477, 18 November 2013); informed consent was obtained from all subjects before examination. All patients furnished written content for these tests and for use of personal data for research purposes. This study has been approved by the Azienda Universitaria Policlinico (AUP) of the Second University of Naples, prior consultation with the ethics committee of the same AUP, which issued the ethics code RDn. 1185 of July 27, 2011, subsequently integrated with Decree 922 October 22, 2013.

\section{Diets}

The diets for each patient were processed in a personalized way. To draw each patient has been practiced BIA (bioelectrical impedance analysis) and then calculated the recommended quantity and quality of food following the RDA (Recommended Daily Allowance) according to the INRAN, (Istituto Nazionale di ricerca per gli alimenti e nutrizione). Recommended diets have considered blood levels of cholesterol starting integer and fractional, glucose, bilirubin, transaminases, uric acid and triglycerides, so the diet that the patient followed for three months was low-calorie, low-salt, and low-fat.

\section{Experiment}

To analyze the biochemical and macular changes of 40 pcs obese, 20 males and 20 females, affected by metabolic syndrome, and which expressed increased macular thickness regularly monitored by biochemical point of view, showed, after a low-calorie diet, low fat and low salt, a remarkable change, recovery from metabolic syndrome and improvement of the alteration of the macular thickness.

Patients were monitored every week with the BIA (bioelectrical impedance analysis), so from time to time by comparing the values of lean body mass (\%), body fat (\%), total water (\%), intra-and extracellular water (\%), basal metabolic rate, BMI and body weight.

\section{Biochemical Analyses}

Serum levels of FT3, FT4 and TSH were measured with the Benvenga and Trimarchi method [8]; all the materials necessary have been furnished by DiaSorin (Saluggia, Italy).

Blood tests for total cholesterol, low density lipoproteins (LDL), higth density lipoproteins (HDL), triglycerides, circulating glucose, uric acid and bilirubin were performed before starting the diet therapy regimen. The same was done at the end of experimental time.

\section{Ocular Coherence Tomography (OCT)}

Every patient underwent a complete ophthalmic examination, only those ones with best corrected visual acuity (BCVA) of 20/20 clear media, refractive error in the range between $-3 \mathrm{D}$ and $+3 \mathrm{D}$ (measured as spherical equivalent), normal retina status in both eyes and no history or evidence of either systemic or ophthalmic disease were included [7].

RTVue-100 Fourier Domain OCT, (Optovue Inc, Fremont, CA, USA), software version 2.0 has been used for macular assessment. The instrument has an axial resolution of $5 \mu \mathrm{m}$ and performs 26000 scans per second.

Each patient was taken through one acquisition session for both eyes (first the right eye then the left), the OCT instruments were operated by the same trained operator. Only OCT scans that were of sufficient quality (signal $\geq 60 \%$ of maximum strength, absence of imaging artifacts, or distortions) were used. Replicates were only taken in case of poor quality OCT scans. Patient's pupils were not dilated, as the device is able to take high quality macular scan in dark room without drugs help.

After the acquisition protocol and scan procedure were explained to them in detail, the subjects were placed in front of the OCT instrument and asked to fixate on an internal fixation target. After the focus was 
Citation: Esposito T, Varriale B, Irregolare C, Lanza M, Perna A, et al. (2015) Correlation between Thyroid Endocrine Status, Change in Body Weight and Macular Thickness in Obese Patients. Gen Med (Los Angel) 3: 206. doi:10.4172/2327-5146.1000206

Page 3 of 5

adjusted and a good central fixation obtained, the scanning process was started.

Thickness maps were calculated with the built-in analysis software of the OCT. Acquisition strategy has been: Macular map $(512 \times 101$ scan pattern, 101 lines; 512 A-scans per line, scan area: $5 \times 5 \mathrm{~mm}$, acquisition time for scan: 2.0 seconds).

All these procedures have been performed before starting the diet and at 3 months follow up.

Also both eyes of every patient has been evaluated, only right eyes have been included in the statistical analysis.

\section{Statistical Analysis}

A one-way ANOVA (Welch's test) model was applied to analyze growth and metabolic variables: $\Upsilon_{\mathrm{ij}}=\mu+\alpha_{\mathrm{I}}+\varepsilon_{\mathrm{ij}}$ where $\mu=$ population average; $\alpha_{\mathrm{i}}=$ treatment effect and $\varepsilon_{\mathrm{ij}}=$ random error. For the correlations in patients treated among biochemical analysis, macular thickness and body parameters were used "Spearman's rank order correlations" is a correlation test nonparametric because the magnitudes being compared were an unequal type (\% vs. physical data of weight for example) and variability. For all tests was considered as the limit of significance set at $\mathrm{p}<0.05(<5 \%)$. For their determination we used the SPSS software.

\section{Results and Discussion}

Generally the metabolic syndrome found in states of obesity (BMI> 30 ), leads to secondary pathologies in different organs [1].

The first goal of our work has been the significative reduction in the concentration of FT3, FT4 and TSH that are fastly reported into the normal range (Figure 1). This appears to be a necessary tool to recovery from the metabolic syndrome they suffer.

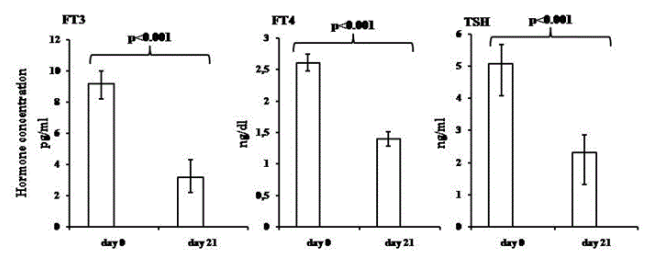

Figure 1: Variation in FT3, FT4 and TSH concentration in obese patients with altered hormone concentration after 21 days of a low calorie diet, low fat and low salt content.

Our data show that patients affected by obesity and high levels of thyroid hormones can achieve a statistically significant macular thickness reduction 3 months after hypo caloric diet (Figures 2 and 3). In particular, the extent of the reduction of macular thickness is inversely correlated only with the values of bilirubin pre (Rs: -0483 , $\mathrm{p}<0.031$ ) and post-diet (Rs: -0461, $\mathrm{p}<0.041$ ) (Figure 4).

We also found significant correlations between the thickness macular detected pre-treatment and post-treatment\% lean mass (Rs: -0606, $\mathrm{p}<0.005$ ), BMI post-treatment (Rs 0.554, $\mathrm{p}<0.011$ ), the $\%$ reduction in BMI (Rs $0.508, \mathrm{p}<0.022)$ and\% reduction in body weight (Rs $0.492, \mathrm{p}<0.027$ ) (Figure 5). Finally, the same significant correlations are also present between the thickness macular detected posttreatment and post-treatment\% lean mass (Rs: $-0580, \mathrm{p}<0.007), \mathrm{BMI}$ post-treatment (Rs $0.583, \mathrm{p}<0.007$ ), the\% reduction in BMI (Rs: 0.452, $\mathrm{p}<0.045$ ) and $\%$ reduction in body weight (Rs 0.459, $\mathrm{p}<0.042$ ) (Figure $6)$.

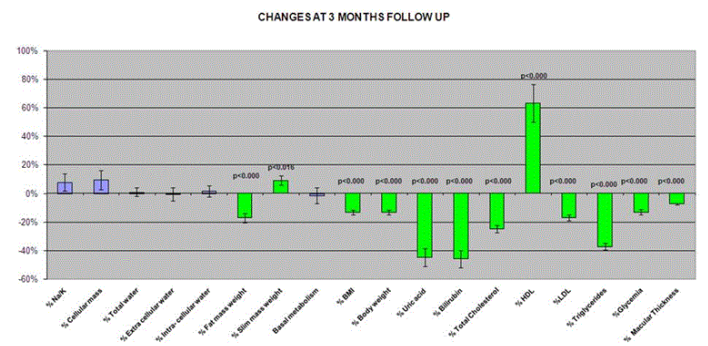

Figure 2: With regard to body parameters measured in patients considered significant variations are related to diet after weight $\%$ fat mass $(-17 \%, \mathrm{p}<0.000), \%$ by weight lean body mass $(+9.0 \%$, $\mathrm{p}<0.016)$, BMI $(-13 \%, \mathrm{p}<0.000)$ and body weight $(-13 \%, \mathrm{p}<0.000)$. In addition, all blood parameters are varied significantly: Ac. uric acid $(-45 \%, \mathrm{p}<0.000)$, bilirubin $(-46 \%, \mathrm{p}<0.000)$, total cholesterol. $(-25 \%, p<0.000)$, HDL $(+63 \%, p<0.000)$, LDL $(-17 \%, p<0.000)$, triglycerides $(-37 \%, \mathrm{p}<0.000)$, and blood glucose $(-13 \% \mathrm{p}<0.000)$. Finally also the reduction of the thickness macular is significant $(-7.5 \%, \mathrm{p}<0.000)$

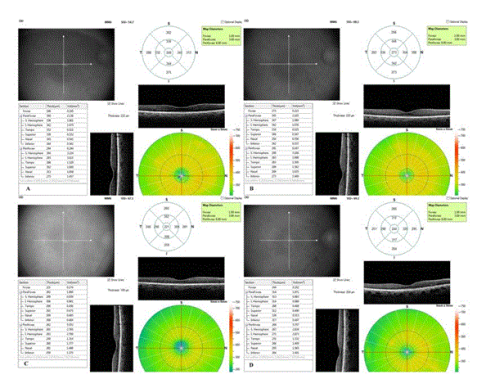

Figure 3: Screenshots of macular thickness with OCT before diet $(A, B)$ and after diet $(C, D)$ in two right eyes of two different patients.
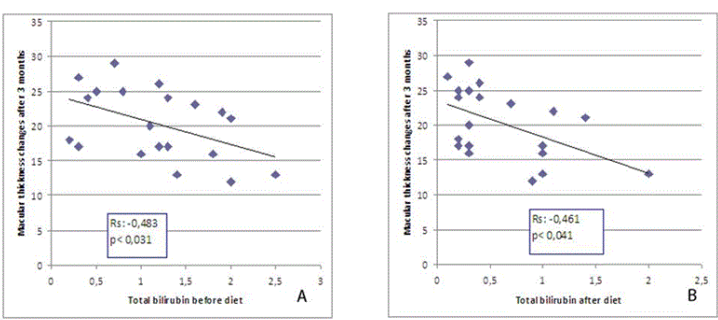

Figure 4: The reduction of macular thickness is inversely correlated only with the values of bilirubin pre (Rs: $-0483, \mathrm{p}<0.031$ ) and postdiet (Rs: -0461, $\mathrm{p}<0.041$ ) 


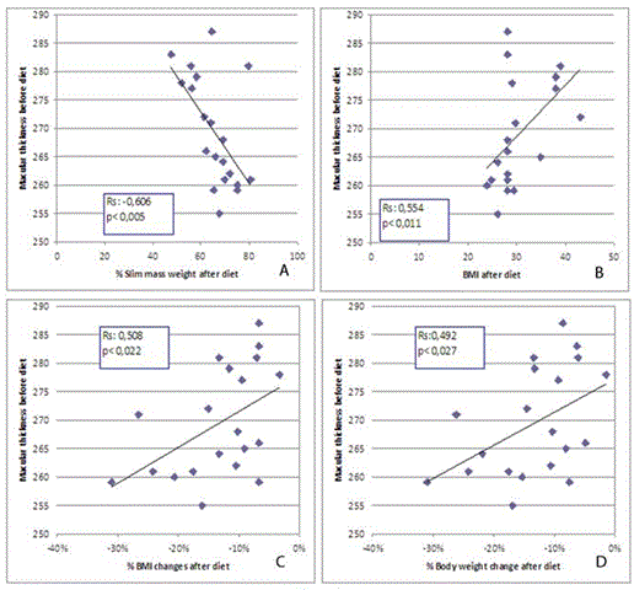

Figure 5: Significant correlations between the macular thickness detected pre-treatment and post-treatment vs. \% lean mass (Rs: $-0606, \mathrm{p}<0.005)$, vs. BMI post-treatment (Rs $0.554, \mathrm{p}<0.011$ ), vs. the $\%$ reduction in BMI (Rs: 0.508, $\mathrm{p}<0.022$ ) and vs. \% reduction in body weight (Rs $0.492, \mathrm{p}<0.027)$

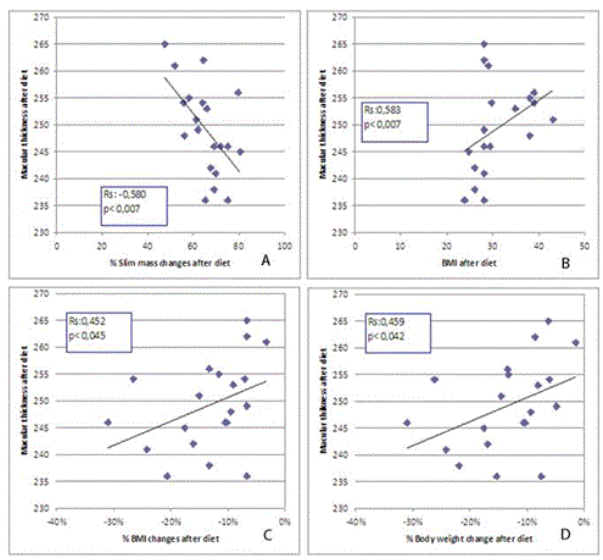

Figure 6: Significant correlations were also found between the macular thickness detected post-treatment and post-treatment \% lean mass (Rs: $-0580, \mathrm{p}<0.007$ ), BMI post-treatment (Rs 0.583, $\mathrm{p}<0.007$ ), the $\%$ of reduction in BMI (Rs: $0.452, \mathrm{p}<0.045)$ and $\%$ reduction in body weight (Rs $0.459, \mathrm{p}<0.042$ ).

Obesity can be defined as a syndrome characterized by an increased risk of morbidity and mortality [9]. Excess body weight is associated to different co-morbidities in a heterogeneous way [1]. An accurate assessment of nutritional status, in its three components: body composition, energy balance and functionality, is the mastermind to set a proper treatment which must be of multidimensional type. The metabolic syndrome associated with obesity itself does not exclude the possibility of the increase in macular thickness. Following the production of pro-inflammatory factors as (TNFa, IL 6, PAI 1, IGF 1) and hormones that modulate various metabolic processes including the regulation of glucose and fatty acid metabolism (FFA, leptin, adiponectin) by the visceral adipose tissue. These pro-inflammatory factors, caused by increased fat mass and cholesterol, could produce the macular stiffening and in turn an alteration of macular microvasculature. Our data show that this enlarged macular thickness can be reduced by hypo caloric diet, probably related to the better physiological status of patients.

\section{Conclusions}

This leads us to conclude that a low calorie diet, low fat and low salt content, in obese patients can reduce the possibility of metabolic syndrome and the risk to develop retinal alterations. This can be explained because the body weight and fat mass lowering could avoid a greater recall of water and/or lipids causing the macular thickening (Figure 7).

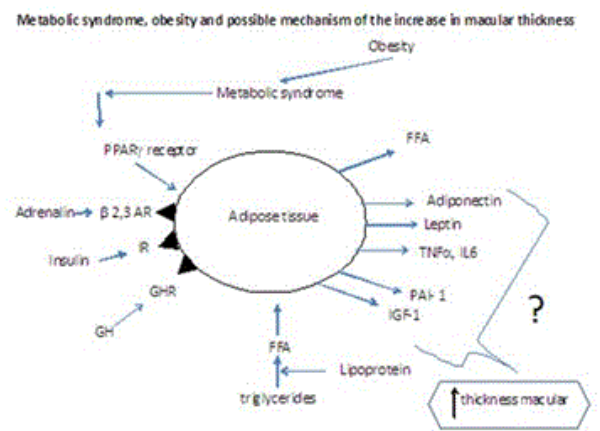

Figure 7: Metabolic syndrome, obesity and possible mechanism of the increase in macular thickness

To our knowledge, this is the first paper studying correlation among body and blood parameters changes versus macular thickness variation in obese patients after 3 months from starting a hypo caloric diet. Low calorie diet, low fat and low salt content, in obese patients can reduce the risk to develop retinal alterations. It could be important to introduce OCT evaluation in obese patients (BMI>30) to better manage both general and ocular obesity related changes. This tool may be important also in early detection of macular alterations in obese patients not necessarily related to diabetes.

\section{References}

1. Aballay LR, Eynard AR, Daz Medel P, Navarro A, Muoz SE (2013) Overweight and obesity: a review of their relationship to metabolic syndrome, cardiovascular disease, and cancer in South America, Nutr Rev 71: 168-179.

2. Lukaski HC (2001) Body mass index, bioelectrical impedance, and body composition. Nutrition 17: 55-56.

3. Wang J, Thornton JC, Bari S, Williamson B, Gallagher D, et al. (2003) Comparisons of waist circumferences measured at 4 sites. Am J Clin Nutr 77: 379-384.

4. Zamboni M, Turcato E, Armellini F, Kahn HS, Zivelonghi A, et al. (1998) Sagittal abdominal diameter as a practical predictor of visceral fat. Int J Obes Relat Metab Disord 22: 655-660.

5. Verga S, Buscemi Le S (1995) obesitÃ primitive e secondarie. La Medicina Internazionale, Raffaello Cortina Editore 8.

6. [No authors listed] (1998) Clinical guidelines on the identification, evaluation, and treatment of overweight and obesity in adults: executive summary. Expert Panel on the Identification, Evaluation, and Treatment of Overweight in Adults. Am J Clin Nutr 68: 899-917. 
Citation: Esposito T, Varriale B, Irregolare C, Lanza M, Perna A, et al. (2015) Correlation between Thyroid Endocrine Status, Change in Body Weight and Macular Thickness in Obese Patients. Gen Med (Los Angel) 3: 206. doi:10.4172/2327-5146.1000206

Page 5 of 5

7. Gooding KM, Tooke JE, von Lany H, Mitra M, Ling R, et al. (2010) Capillary pressure may predict preclinical changes in the eye.

9. Brown J (2012) Managing obesity in adults. Nurs Times 108: 18-19. Diabetologia 53: 2029-2035.

8. Benvenga S, Trimarchi F (2008) Changed presentation of Hashimoto's thyroiditis in North-Eastern Sicily and Calabria (Southern Italy) based on a 31-year experience. Thyroid 18: 429-441. 